\title{
Stability of the Drygas Functional Equation on Restricted Domain
}

\author{
Magdalena Piszczek and Joanna Szczawińska
}

\begin{abstract}
We study the stability of the Drygas functional equation on a restricted domain. The main tool used in the proofs is the fixed point theorem for functional spaces.
\end{abstract}

Mathematics Subject Classification. 39B82, 47H10, 47J20.

Keywords. Drygas equation, stability, fixed point theorem.

\section{Introduction}

We say that a function $f: \mathbb{R} \rightarrow \mathbb{R}$ satisfies the Drygas equation iff

$$
f(x+y)+f(x-y)=2 f(x)+f(y)+f(-y), \quad x, y \in \mathbb{R} .
$$

The above equation was introduced in [3] in order to obtain a characterization of the quasi-inner-product spaces. Ebanks, Kannappan and Sahoo in [4] have obtained the general solution of the Eq. (1) as

$$
f(x)=a(x)+q(x), \quad x \in \mathbb{R},
$$

where $a: \mathbb{R} \rightarrow \mathbb{R}$ is an additive function and $q: \mathbb{R} \rightarrow \mathbb{R}$ is a quadratic function, i.e. $q$ satisfies the quadratic functional equation

$$
q(x+y)+q(x-y)=2 q(x)+2 q(y), \quad x, y \in \mathbb{R} .
$$

A set-valued version of Eq. (1) was considered by Smajdor in [8].

The stability in the Hyers-Ulam sense of the Drygas equation has been investigated by Jung and Sahoo in [6]. They have proved that if a function $f: X \rightarrow Y$, where $X$ is a real vector space and $Y$ is a Banach space satisfies the inequality

$$
\|f(x+y)+f(x-y)-2 f(x)-f(y)-f(-y)\| \leq \varepsilon, \quad x, y \in X
$$


for same $\varepsilon>0$, then there exists a unique additive function $a: X \rightarrow Y$ and and a unique quadratic function $q: X \rightarrow Y$ such that

$$
\|f(x)-a(x)-q(x)\| \leq \frac{25}{3} \varepsilon, \quad x \in X .
$$

Their result was improved first by Yang in [9] and later by Sikorska in [7]. In the case when $X$ is an Abelian group they obtained sharper bounds: $\frac{3}{2} \varepsilon$ and $\varepsilon$ respectively instead of $\frac{25}{3} \varepsilon$ (cf. Proposition 1 in [9] and Theorem 3.2 in [7]). The stability and solution of the Drygas equation under some additional conditions was also studied by Forti and Sikorska in [5] in the case when $X$ and $Y$ are amenable groups.

In the paper we present the stability results for the Drygas equation on restricted domain. Let $X$ be a nonempty subset of a normed space and $Y$ be a normed space. We say that a function $f: X \rightarrow Y$ satisfies the Drygas functional equation on $X$ if

$$
f(x+y)+f(x-y)=2 f(x)+f(y)+f(-y), \quad x, y \in X, x+y, x-y \in X .
$$

One of the method of the proof is based on a fixed point result that can be derived from [1] (Theorem 1). To present it we need the following three hypothesis:

(H1) $X$ is a nonempty set, $Y$ is a Banach space, $f_{1}, \ldots, f_{k}: X \rightarrow X$ and $L_{1}, \ldots, L_{k}: X \rightarrow \mathbb{R}_{+}$are given.

(H2) $\mathcal{T}: Y^{X} \rightarrow Y^{X}$ is an operator satisfying the inequality

$$
\|\mathcal{T} \xi(x)-\mathcal{T} \mu(x)\| \leq \sum_{i=1}^{k} L_{i}(x)\left\|\xi\left(f_{i}(x)\right)-\mu\left(f_{i}(x)\right)\right\|
$$

for all $\xi, \mu \in Y^{X}, x \in X$.

(H3) $\Lambda: \mathbb{R}_{+}{ }^{X} \rightarrow \mathbb{R}_{+}{ }^{X}$ is defined by

$$
\Lambda \delta(x):=\sum_{i=1}^{k} L_{i}(x) \delta\left(f_{i}(x)\right), \quad \delta \in \mathbb{R}_{+}{ }^{X}, x \in X .
$$

Now we are in a position to present the above mentioned fixed point theorem.

Theorem 1. Let hypotheses (H1)-(H3) be valid and functions $\varepsilon: X \rightarrow \mathbb{R}_{+}$and $\varphi: X \rightarrow Y$ fulfil the following two conditions

$$
\begin{aligned}
& \|\mathcal{T} \varphi(x)-\varphi(x)\| \leq \varepsilon(x), \quad x \in X, \\
& \varepsilon^{*}(x):=\sum_{n=0}^{\infty} \Lambda^{n} \varepsilon(x)<\infty, \quad x \in X .
\end{aligned}
$$

Then there exists a unique fixed point $\psi$ of $\mathcal{T}$ with

$$
\|\varphi(x)-\psi(x)\| \leq \varepsilon^{*}(x), \quad x \in X .
$$


Moreover,

$$
\psi(x):=\lim _{n \rightarrow \infty} \mathcal{T}^{n} \varphi(x), \quad x \in X .
$$

Throughout the paper $\mathbb{N}_{0}$ denotes the set of all non-negative integers.

\section{Stability Results}

Theorem 2. Let $X$ be a subset with 0 of a normed space, $Y$ be a Banach space and $c \geq 0$. Assume that $p>0$ and a function $f: X \rightarrow Y$ satisfies

$$
\|f(x+y)+f(x-y)-2 f(x)-f(y)-f(-y)\| \leq c\left(\|x\|^{p}+\|y\|^{p}\right)
$$

for all $x, y \in X$ such that $x+y, x-y \in X$.

(1) If $p>2$ and $-x, \frac{x}{2} \in X$ for all $x \in X$, then there exists a function $g: X \rightarrow Y$ satisfying the Drygas equation on $X$ such that

$$
\|f(x)-g(x)\| \leq \frac{2 c}{2^{p}-4}\|x\|^{p}, \quad x \in X .
$$

(2) If $0<p<1$ and $-x, 2 x \in X$ for all $x \in X$, then there exists a function $g: X \rightarrow Y$ satisfying the Drygas equation on $X$ such that

$$
\|f(x)-g(x)\| \leq \frac{2 c}{2-2^{p}}\|x\|^{p}, \quad x \in X .
$$

(3) If $1<p<2$ and $-x, \frac{1}{2} x, 2 x \in X$ for all $x \in X$, then there exists $a$ function $g: X \rightarrow Y$ satisfying the Drygas equation on $X$ such that

$$
\|f(x)-g(x)\| \leq\left(\frac{2 c}{4-2^{p}}+\frac{2 c}{2^{p}-2}\right)\|x\|^{p}, \quad x \in X .
$$

Moreover, $g$ is the unique solution of the Eq. (3) such that $\|f(x)-g(x)\| \leq$ $M\|x\|^{p}$ for all $x \in X$ and some $M \geq 0$.

Proof. First observe that the inequality (4) clearly forces $f(0)=0$.

(1) Replacing $x$ and $y$ by $\frac{x}{2}$ in (4) we obtain

$$
\left\|f(x)-3 f\left(\frac{x}{2}\right)-f\left(-\frac{x}{2}\right)\right\| \leq \frac{2 c}{2^{p}}\|x\|^{p}, \quad x \in X .
$$

Consider functions $\mathcal{T}: Y^{X} \rightarrow Y^{X}$ and $\varepsilon: X \rightarrow \mathbb{R}_{+}$given as follows

$$
\mathcal{T} \xi(x)=3 \xi\left(\frac{x}{2}\right)+\xi\left(-\frac{x}{2}\right), \quad x \in X, \xi \in Y^{X}
$$

and

$$
\varepsilon(x)=\frac{2 c}{2^{p}}\|x\|^{p}, \quad x \in X .
$$

The inequality (5) now becomes

$$
\|\mathcal{T} f(x)-f(x)\| \leq \varepsilon(x), \quad x \in X
$$


For every $\xi, \mu \in Y^{X}$ and $x \in X$

$$
\|\mathcal{T} \xi(x)-\mathcal{T} \mu(x)\| \leq 3\left\|\xi\left(\frac{x}{2}\right)-\mu\left(\frac{x}{2}\right)\right\|+\left\|\xi\left(-\frac{x}{2}\right)-\mu\left(-\frac{x}{2}\right)\right\|,
$$

so $\mathcal{T}$ satisfies the inequality (H2) with $f_{1}(x)=\frac{x}{2}, f_{2}(x)=-\frac{x}{2}, L_{1}(x)=3$, $L_{2}(x)=1, x \in X$. By (H3), the operator $\Lambda: \mathbb{R}_{+}^{X} \rightarrow \mathbb{R}_{+}^{X}$ is given by

$$
\Lambda \eta(x)=3 \eta\left(\frac{x}{2}\right)+\eta\left(-\frac{x}{2}\right), \quad x \in X, \eta \in \mathbb{R}_{+}^{X} .
$$

In particular

$$
\Lambda \varepsilon(x)=4 \varepsilon\left(\frac{x}{2}\right)=\frac{4}{2^{p}} \varepsilon(x), \quad x \in X .
$$

Since $\Lambda$ is linear, we can prove by induction

$$
\Lambda^{n} \varepsilon(x)=\left(\frac{4}{2^{p}}\right)^{n} \varepsilon(x), \quad x \in X, n \in \mathbb{N}_{0} .
$$

As $p>2$ we have $\frac{4}{2^{p}}<1$. Consequently the series $\sum_{n=0}^{\infty} \Lambda^{n} \varepsilon(x)$ is convergent for every $x \in X$ and

$$
\varepsilon^{*}(x)=\sum_{n=0}^{\infty} \Lambda^{n} \varepsilon(x)=\sum_{n=0}^{\infty}\left(\frac{4}{2^{p}}\right)^{n} \varepsilon(x)=\frac{2^{p}}{2^{p}-4} \varepsilon(x)=\frac{2 c}{2^{p}-4}\|x\|^{p}, \quad x \in X .
$$

By Theorem 1, there exists a function $g: X \rightarrow Y$ such

$$
\begin{aligned}
& g(x)=\lim _{n \rightarrow \infty} \mathcal{T}^{n} f(x), \quad x \in X, \\
& g(x)=3 g\left(\frac{x}{2}\right)+g\left(-\frac{x}{2}\right), \quad x \in X
\end{aligned}
$$

and

$$
\|f(x)-g(x)\| \leq \frac{2 c}{2^{p}-4}\|x\|^{p}, \quad x \in X .
$$

Next we prove that $g$ satisfies the Drygas equation. Observe first that if a function $h: X \rightarrow Y$ satisfies the inequality

$$
\|h(x+y)+h(x-y)-2 h(x)-h(y)-h(-y)\| \leq M\left(\|x\|^{p}+\|y\|^{p}\right)
$$

for all $x, y \in X$ such that $x+y, x-y \in X$ and some $M>0$, then

$$
\|\mathcal{T} h(x+y)+\mathcal{T} h(x-y)-2 \mathcal{T} h(x)-\mathcal{T} h(y)-\mathcal{T} h(-y)\| \leq \frac{4 M}{2^{p}}\left(\|x\|^{p}+\|y\|^{p}\right),
$$


for $x, y \in X$ satisfying $x+y, x-y \in X$. Indeed, fix $h: X \rightarrow Y$ and assume (6). Then

$$
\begin{aligned}
\mathcal{T} h(x+y)+\mathcal{T} h(x-y)-2 \mathcal{T} h(x)-\mathcal{T} h(y)-\mathcal{T} h(-y) \\
=3\left(h\left(\frac{x+y}{2}\right)+h\left(\frac{x-y}{2}\right)-2 h\left(\frac{x}{2}\right)-h\left(\frac{y}{2}\right)-h\left(-\frac{y}{2}\right)\right) \\
\quad+\left(h\left(-\frac{x+y}{2}\right)+h\left(-\frac{x-y}{2}\right)-2 h\left(-\frac{x}{2}\right)-h\left(-\frac{y}{2}\right)-h\left(\frac{y}{2}\right)\right)
\end{aligned}
$$

for all $x, y \in X, x+y, x-y \in X$. Hence

$$
\begin{aligned}
\|\mathcal{T} h(x+y)+\mathcal{T} h(x-y)-2 \mathcal{T} h(x)-\mathcal{T} h(y)-\mathcal{T} h(-y)\| \\
\leq 3\left\|h\left(\frac{x+y}{2}\right)+h\left(\frac{x-y}{2}\right)-2 h\left(\frac{x}{2}\right)-h\left(\frac{y}{2}\right)-h\left(-\frac{y}{2}\right)\right\| \\
\quad+\left\|h\left(-\frac{x+y}{2}\right)+h\left(-\frac{x-y}{2}\right)-2 h\left(-\frac{x}{2}\right)-h\left(-\frac{y}{2}\right)-h\left(\frac{y}{2}\right)\right\| \\
\leq 3 M\left(\left\|\frac{x}{2}\right\|^{p}+\left\|\frac{y}{2}\right\|^{p}\right)+M\left(\left\|-\frac{x}{2}\right\|^{p}+\left\|-\frac{y}{2}\right\|^{p}\right) \\
=\frac{4 M}{2^{p}}\left(\|x\|^{p}+\|y\|^{p}\right) .
\end{aligned}
$$

Consequently, proceeding by induction we get that if a function $h: X \rightarrow Y$ satisfies the inequality $(6)$, then

$$
\begin{aligned}
& \left\|\mathcal{T}^{n} h(x+y)+\mathcal{T}^{n} h(x-y)-2 \mathcal{T}^{n} h(x)-\mathcal{T}^{n} h(y)-\mathcal{T}^{n} h(-y)\right\| \\
& \quad \leq M\left(\frac{4}{2^{p}}\right)^{n}\left(\|x\|^{p}+\|y\|^{p}\right)
\end{aligned}
$$

for all $n \in \mathbb{N}_{0}$ and $x, y \in X, x+y, x-y \in X$. On account of the above observation and (4)

$$
\begin{aligned}
& \left\|\mathcal{T}^{n} f(x+y)+\mathcal{T}^{n} f(x-y)-2 \mathcal{T}^{n} f(x)-\mathcal{T}^{n} f(y)-\mathcal{T}^{n} f(-y)\right\| \\
& \quad \leq c\left(\frac{4}{2^{p}}\right)^{n}\left(\|x\|^{p}+\|y\|^{p}\right)
\end{aligned}
$$

for every $n \in \mathbb{N}_{0}$ and $x, y \in X$ such that $x+y, x-y \in X$. Letting $n \rightarrow \infty$ we get

$g(x+y)+g(x-y)=2 g(x)+g(y)+g(-y), \quad x, y \in X, x+y, x-y \in X$.

(2) The idea of the proof is the same as before so we only give a sketch. Replacing $y$ by $x$ in (4) we obtain

$$
\left\|\frac{1}{3} f(2 x)-\frac{1}{3} f(-x)-f(x)\right\| \leq \frac{2 c}{3}\|x\|^{p}, \quad x \in X .
$$


Let functions $\mathcal{T}: Y^{X} \rightarrow Y^{X}$ and $\varepsilon: X \rightarrow \mathbb{R}_{+}$be define by formulas

$$
\mathcal{T} \xi(x)=\frac{1}{3} \xi(2 x)-\frac{1}{3} \xi(-x), \quad x \in X, \xi \in Y^{X}
$$

and

$$
\varepsilon(x)=\frac{2 c}{3}\|x\|^{p}, \quad x \in X .
$$

The inequality (7) takes now the form

$$
\|\mathcal{T} f(x)-f(x)\| \leq \varepsilon(x), \quad x \in X
$$

Obviously $\mathcal{T}$ satisfies the inequality $(\mathrm{H} 2)$ with $f_{1}(x)=2 x, f_{2}(x)=-x, L_{1}(x)=$ $L_{2}(x)=\frac{1}{3}, x \in X$. The operator $\Lambda: \mathbb{R}_{+}^{X} \rightarrow \mathbb{R}_{+}^{X}$ is given by

In particular

$$
\Lambda \eta(x)=\frac{1}{3} \eta(2 x)+\frac{1}{3} \eta(-x), \quad x \in X, \eta \in \mathbb{R}_{+}^{X} .
$$

$$
\Lambda \varepsilon(x)=\frac{1}{3} \varepsilon(2 x)+\frac{1}{3} \varepsilon(-x)=\frac{2^{p}+1}{3} \varepsilon(x), \quad x \in X .
$$

Proceeding by induction, we obtain

$$
\Lambda^{n} \varepsilon(x)=\left(\frac{2^{p}+1}{3}\right)^{n} \varepsilon(x), \quad x \in X, n \in \mathbb{N}_{0} .
$$

Since $p<1, \frac{2^{p}+1}{3}<1$, so the series $\sum_{n=0}^{\infty} \Lambda^{n} \varepsilon(x)$ is convergent for every $x \in X$ and

$$
\varepsilon^{*}(x)=\sum_{n=0}^{\infty} \Lambda^{n} \varepsilon(x)=\frac{3}{2-2^{p}} \varepsilon(x)=\frac{2 c}{2-2^{p}}\|x\|^{p}, \quad x \in X .
$$

By Theorem 1, there exists a function $g: X \rightarrow Y$ such

$$
\begin{aligned}
& g(x)=\lim _{n \rightarrow \infty} \mathcal{T}^{n} f(x), \quad x \in X, \\
& g(x)=\frac{1}{3} g(2 x)-\frac{1}{3} g(-x), \quad x \in X
\end{aligned}
$$

and

$$
\|f(x)-g(x)\| \leq \frac{2 c}{2-2^{p}}\|x\|^{p}, \quad x \in X .
$$

A trivial verification shows that

$$
\begin{aligned}
& \left\|\mathcal{T}^{n} f(x+y)+\mathcal{T}^{n} f(x-y)-2 \mathcal{T}^{n} f(x)-\mathcal{T}^{n} f(y)-\mathcal{T}^{n} f(-y)\right\| \\
& \quad \leq\left(\frac{2^{p}+1}{3}\right)^{n} c\left(\|x\|^{p}+\|y\|^{p}\right)
\end{aligned}
$$

for every $n \in \mathbb{N}_{0}$ and $x, y \in X$ satisfying $x+y, x-y \in X$. Hence, letting $n \rightarrow \infty$ we obtain

$g(x+y)+g(x-y)=2 g(x)+g(y)+g(-y), \quad x, y \in X, x+y, x-y \in X$. 
(3) In this case let $f_{e}: X \rightarrow Y$ and $f_{o}: X \rightarrow Y$ be the even and the odd part of the function $f$, respectively. That means $f_{e}(x)=\frac{f(x)+f(-x)}{2}, f_{o}(x)=$ $\frac{f(x)-f(-x)}{2}$ for $x \in X$ and $f=f_{e}+f_{o}$. It is easy to see that $f(0)=f_{e}(0)=$ $f_{o}(0)=0$. It follows that

$$
\begin{aligned}
\| f_{e}(x+ & y)+f_{e}(x-y)-2 f_{e}(x)-f_{e}(y)-f_{e}(-y) \| \\
= & \frac{1}{2} \| f(x+y)+f(-x-y)+f(x-y)+f(-x+y) \\
& \quad-2 f(x)-2 f(-x)-f(y)-f(-y)-f(-y)-f(y) \| \\
\leq & \frac{1}{2}(\|f(x+y)+f(x-y)-2 f(x)-f(y)-f(-y)\| \\
& \quad+\|f(-x-y)+f(-x+y)-2 f(-x)-f(-y)-f(y)\|) \\
\leq & c\left(\|x\|^{p}+\|y\|^{p}\right)
\end{aligned}
$$

and analogously

$$
\left\|f_{o}(x+y)+f_{o}(x-y)-2 f_{o}(x)-f_{o}(y)-f_{o}(-y)\right\| \leq c\left(\|x\|^{p}+\|y\|^{p}\right)
$$

for every $x, y \in X$ such that $x+y, x-y \in X$. Hence $f_{e}, f_{o}$ satisfy the inequality (4).

Replace $y$ by $x$ in (4). By the evenness of $f_{e}$,

$$
\left\|f_{e}(2 x)-4 f_{e}(x)\right\| \leq 2 c\|x\|^{p}, \quad x \in X
$$

which gives

$$
\left\|f_{e}(x)-\frac{f_{e}(2 x)}{4}\right\| \leq \frac{1}{2} c\|x\|^{p}, \quad x \in X .
$$

Let

$$
\begin{array}{ll}
\mathcal{T} \xi(x)=\frac{\xi(2 x)}{4}, & \xi \in Y^{X}, \quad x \in X, \\
\Lambda \delta(x)=\frac{\delta(2 x)}{4}, & \delta \in \mathbb{R}_{+}^{X}, \quad x \in X
\end{array}
$$

and $\varepsilon(x)=\frac{1}{2} c\|x\|^{p}, x \in X$. By Theorem 1, there exists a function $g_{e}: X \rightarrow Y$ such that

$$
\begin{aligned}
& g_{e}(x)=\lim _{n \rightarrow \infty} \mathcal{T}^{n} f_{e}(x), \quad x \in X, \\
& g_{e}(x)=\frac{g_{e}(2 x)}{4}, \quad x \in X
\end{aligned}
$$

and

$$
\left\|f_{e}(x)-g_{e}(x)\right\| \leq \frac{2 c}{4-2^{p}}\|x\|^{p}, \quad x \in X
$$


Moreover,

$$
\begin{aligned}
& \left\|\mathcal{T}^{n} f_{e}(x+y)+\mathcal{T}^{n} f_{e}(x-y)-2 \mathcal{T}^{n} f_{e}(x)-\mathcal{T}^{n} f_{e}(y)-\mathcal{T}^{n} f_{e}(-y)\right\| \\
& \quad \leq\left(\frac{2^{p}}{4}\right)^{n} c\left(\|x\|^{p}+\|y\|^{p}\right),
\end{aligned}
$$

for every $n \in \mathbb{N}_{0}$ and $x, y \in X$ satisfying $x+y, x-y \in X$. Hence $g_{e}$ satisfies the Drygas equation.

In the same way, replacing $y$ by $x$ in (4) and using the oddness of $f_{o}$, we obtain

$$
\left\|f_{o}(2 x)-2 f_{o}(x)\right\| \leq 2 c\|x\|^{p}, \quad x \in X
$$

which with $x$ replacing by $\frac{x}{2}$ yields

$$
\left\|f_{o}(x)-2 f_{o}\left(\frac{x}{2}\right)\right\| \leq \frac{2}{2^{p}} c\|x\|^{p}, \quad x \in X .
$$

Define now

$$
\begin{array}{ll}
\mathcal{T} \xi(x)=2 \xi\left(\frac{x}{2}\right), & \xi \in Y^{X}, x \in X, \\
\Lambda \delta(x)=2 \delta\left(\frac{x}{2}\right), & \delta \in \mathbb{R}_{+}^{X}, x \in X
\end{array}
$$

and $\varepsilon(x)=\frac{2}{2^{p}} c\|x\|^{p}, x \in X$. By Theorem 1, there exists a function $g_{o}: X \rightarrow Y$ such that

$$
\begin{aligned}
& g_{o}(x)=\lim _{n \rightarrow \infty} \mathcal{T}^{n} f_{o}(x), \quad x \in X, \\
& g_{o}(x)=2 g_{o}\left(\frac{x}{2}\right), \quad x \in X
\end{aligned}
$$

and

$$
\left\|f_{o}(x)-g_{o}(x)\right\| \leq \frac{2 c}{2^{p}-2}\|x\|^{p}, \quad x \in X .
$$

By

$$
\begin{aligned}
& \left\|\mathcal{T}^{n} f_{o}(x+y)+\mathcal{T}^{n} f_{o}(x-y)-2 \mathcal{T}^{n} f_{o}(x)-\mathcal{T}^{n} f_{o}(y)-\mathcal{T}^{n} f_{o}(-y)\right\| \\
& \quad \leq\left(\frac{2}{2^{p}}\right)^{n} c\left(\|x\|^{p}+\|y\|^{p}\right), \quad n \in \mathbb{N}_{0}, x, y \in X, x+y, x-y \in X,
\end{aligned}
$$

$g_{o}$ satisfies the Drygas equation. Thus $g=g_{e}+g_{o}$ also satisfies the Drygas equation and

$$
\|f(x)-g(x)\| \leq \frac{2 c}{4-2^{p}}\|x\|^{p}+\frac{2 c}{2^{p}-2}\|x\|^{p}, \quad x \in X .
$$


It remains to prove the uniqueness of the function $g$. We show the case $p>2$ in details. Let us assume that functions $g_{1}, g_{2}: X \rightarrow Y$ fulfill the Drygas equation on $X$ and

$$
\left\|f(x)-g_{i}(x)\right\| \leq M_{i}\|x\|^{p}, \quad x \in X
$$

for some $M_{i} \geq 0, i=1,2$. Hence $\left\|g_{1}(x)-g_{2}(x)\right\| \leq\left(M_{1}+M_{2}\right)\|x\|^{p}, x \in X$. Since $g_{1}, g_{2}$ satisfy the Drygas equation,

$$
g_{i}(x)=3 g_{i}\left(\frac{x}{2}\right)+g_{i}\left(-\frac{x}{2}\right), \quad x \in X, i=1,2 .
$$

Thus

$$
\begin{aligned}
\left\|g_{1}(x)-g_{2}(x)\right\| & \leq 3\left\|g_{1}\left(\frac{x}{2}\right)-g_{2}\left(\frac{x}{2}\right)\right\|+\left\|g_{1}\left(-\frac{x}{2}\right)-g_{2}\left(-\frac{x}{2}\right)\right\| \\
& \leq \frac{4}{2^{p}}\left(M_{1}+M_{2}\right)\|x\|^{p}
\end{aligned}
$$

for all $x \in X$. It is easy to check that,

$$
\left\|g_{1}(x)-g_{2}(x)\right\| \leq\left(\frac{4}{2^{p}}\right)^{n}\left(M_{1}+M_{2}\right)\|x\|^{p}, \quad x \in X, n \in \mathbb{N}_{0} .
$$

Letting $n$ to $\infty$ we obtain

$$
g_{1}(x)=g_{2}(x), \quad x \in X
$$

The proofs of the other cases runs as before.

The following examples show that the assumptions putting on the set $X$ can not be omitted.

Example 3. Let $p>2, X=(-\infty,-1] \cup\{0\} \cup[1, \infty)$ and $f(x)=|x|, x \in X$. Then

$$
|f(x+y)+f(x-y)-2 f(x)-f(y)-f(-y)| \leq 2\left(|x|^{p}+|y|^{p}\right)
$$

for all $x, y \in X$ such that $x+y, x-y \in X$. Consider functions $g_{a}: X \rightarrow \mathbb{R}$ given by $g_{a}(x)=a x^{2}, x \in X$, where $a$ is any real constant. The functions $g_{a}$ obviously satisfy the Drygas equation and

$$
\left|f(x)-g_{a}(x)\right| \leq|x|^{p}, \quad x \in X
$$

for all $a \in[0,1]$.

Example 4. Let $p \in(0,1), X=[-1,1]$ and $f(x)=x^{3}, x \in X$. Then for all $x, y \in X$ such that $x+y, x-y \in X$

$$
|f(x+y)+f(x-y)-2 f(x)-f(y)-f(-y)| \leq 6\left(|x|^{p}+|y|^{p}\right) .
$$

Every function $g_{a}: X \rightarrow \mathbb{R}$ given by $g_{a}(x)=a x, x \in X$ with $a \in \mathbb{R}$ satisfies the Drygas equation and

$$
\left|f(x)-g_{a}(x)\right| \leq|x|^{p}, \quad x \in X
$$

for all $a \in[0,1]$. 
Example 5. Let $1<p<2, X=(-\infty,-1] \cup\{0\} \cup[1, \infty)$ and $f(x)=\frac{1}{2}(|x|+$ $x), x \in X$. Then

$$
|f(x+y)+f(x-y)-2 f(x)-f(y)-f(-y)| \leq|x|^{p}+|y|^{p}
$$

for all $x, y \in X$ such that $x+y, x-y \in X$. Functions $g_{a}: X \rightarrow \mathbb{R}$ given by $g_{a}(x)=a x, x \in X$ are solutions of the Drygas equation and

$$
\left|f(x)-g_{a}(x)\right| \leq|x|^{p}, \quad x \in X
$$

for all $a \in[0,1]$.

By the same method, we can also obtain the stability result for $p=0$, but in order to obtain the best known bound we have to make more technical substitutions. The idea is adapted from [7].

Theorem 6. Let $X$ be such a subset of an Abelian group that $0,-x, 2 x, 3 x \in X$ for all $x \in X, Y$ a Banach space and $c \geq 0$. If a function $f: X \rightarrow Y$ satisfies

$$
\|f(x+y)+f(x-y)-2 f(x)-f(y)-f(-y)\| \leq c
$$

for all $x, y \in X$ with $x+y, x-y \in X$, then there exists a function $g: X \rightarrow Y$ satisfying the Drygas equation on $X$ such that

$$
\|f(x)-g(x)\| \leq c, \quad x \in X .
$$

Moreover, $g$ is the unique function satisfying equation (3), such that $\|f(x)-g(x)\| \leq M, x \in X$ for some $M \geq 0$.

Proof. Replace $(x, y)$ in $(8)$ by $(2 x, x)$, next by $(x, 2 x),(-x,-2 x)$ and $(x, x)$ (cf. the proof of Theorem 3.2 in [7]). Then

$$
\begin{array}{r}
\|f(3 x)-2 f(2 x)-f(-x)\| \leq c, \\
\|f(3 x)+f(-x)-2 f(x)-f(2 x)-f(-2 x)\| \leq c, \\
\|-f(-3 x)-f(x)+2 f(-x)+f(-2 x)+f(2 x)\| \leq c, \\
\|f(2 x)+f(0)-3 f(x)-f(-x)\| \leq c,
\end{array}
$$

for $x \in X$. Which with $\|f(0)\| \leq \frac{c}{2}$ give

$$
\|2 f(3 x)-f(-3 x)-9 f(x)\| \leq 6 c, \quad x \in X,
$$

whence

$$
\left\|f(x)-\frac{2}{9} f(3 x)+\frac{1}{9} f(-3 x)\right\| \leq \frac{2}{3} c, \quad x \in X .
$$

Let functions $\mathcal{T}: Y^{X} \rightarrow Y^{X}$ and $\varepsilon: X \rightarrow \mathbb{R}_{+}$be defined as follows

$$
\mathcal{T} \xi(x)=\frac{2}{9} \xi(3 x)-\frac{1}{9} \xi(-3 x), \quad x \in X, \xi \in Y^{X}
$$

and

$$
\varepsilon(x)=\frac{2}{3} c, \quad x \in X
$$


The inequality (9) now takes the form

$$
\|\mathcal{T} f(x)-f(x)\| \leq \varepsilon(x), \quad x \in X .
$$

As before, using Theorem 1, there exists a function $g: X \rightarrow Y$ such that

$$
\begin{aligned}
& g(x)=\lim _{n \rightarrow \infty} \mathcal{T}^{n}(x), \quad x \in X, \\
& g(x)=\frac{2}{9} g(3 x)-\frac{1}{9} g(-3 x), \quad x \in X
\end{aligned}
$$

and

$$
\|f(x)-g(x)\| \leq c, \quad x \in X .
$$

In the same manner as in the proofs of Theorem 2 we show that $g$ satisfies the Drygas equation and $g$ is unique.

\section{Nonstability Results}

In this section we show that for $p \in\{1,2\}$ the Drygas equation is not stable. The idea of the construction of the examples comes from the paper [2].

Example 7. Let $\phi: \mathbb{R} \rightarrow \mathbb{R}$ be defined as

$$
\phi(x)= \begin{cases}-\alpha, & x \leq-1, \\ \alpha x, & -1<x<1, \\ \alpha, & 1 \leq x,\end{cases}
$$

where $\alpha>0$. The function $f: \mathbb{R} \rightarrow \mathbb{R}$ given by

$$
f(x)=\sum_{n=0}^{\infty} \frac{\phi\left(2^{n} x\right)}{2^{n}}, \quad x \in \mathbb{R}
$$

satisfies

$$
|f(x+y)+f(x-y)-2 f(x)-f(y)-f(-y)| \leq 8 \alpha(|x|+|y|),
$$

but there exist no pair $(g, k)$ of a function $g: \mathbb{R} \rightarrow \mathbb{R}$ satisfying the Drygas equation and a constant $k \geq 0$ such that

$$
|f(x)-g(x)| \leq k|x|, \quad x \in \mathbb{R} .
$$

Proof. We observe that $f$ is odd and bounded by $2 \alpha$. Now, we show that (10) holds. For $x=y=0$ and $x, y \in \mathbb{R}$ such that $|x|+|y| \geq 1$ it is obvious. Consider the case $0<|x|+|y|<1$. There exists $N \in \mathbb{N}$ such that

$$
\frac{1}{2^{N}} \leq|x|+|y|<\frac{1}{2^{N-1}} .
$$

Then $\left|2^{N-1} x\right|<1,\left|2^{N-1} y\right|<1,\left|2^{N-1}(x+y)\right|<1,\left|2^{N-1}(x-y)\right|<1$. Hence

$$
2^{n} x, 2^{n} y, 2^{n}(x+y), 2^{n}(x-y) \in(-1,1) \quad \text { for } n=0,1, \ldots, N-1 .
$$


By the definition of $f$,

$$
\begin{aligned}
\mid f(x+ & y)+f(x-y)-2 f(x)-f(y)-f(-y) \mid \\
& =\left|\sum_{n=0}^{\infty} \frac{\phi\left(2^{n}(x+y)\right)}{2^{n}}+\sum_{n=0}^{\infty} \frac{\phi\left(2^{n}(x-y)\right)}{2^{n}}-2 \sum_{n=0}^{\infty} \frac{\phi\left(2^{n} x\right)}{2^{n}}\right| \\
& \leq 4 \alpha \sum_{n=N}^{\infty} \frac{1}{2^{n}}=8 \alpha \frac{1}{2^{N}} \\
& \leq 8 \alpha(|x|+|y|) .
\end{aligned}
$$

Assume that there exist a function $g: \mathbb{R} \rightarrow \mathbb{R}$ satisfying the Drygas equation and a constant $k \geq 0$ such that

$$
|f(x)-g(x)| \leq k|x|, \quad x \in \mathbb{R} .
$$

Since $g$ fulfills the Drygas equation, there exist an additive function $h: \mathbb{R} \rightarrow \mathbb{R}$ and a quadratic function $q: \mathbb{R} \rightarrow \mathbb{R}$ such that $g(x)=h(x)+q(x), x \in \mathbb{R}$. Whence, as $f$ is bounded by $2 \alpha$, we have

$$
|h(x)+q(x)| \leq k|x|+2 \alpha, \quad x \in \mathbb{R} .
$$

In particular,

$$
|h(n x)+q(n x)| \leq k|n x|+2 \alpha, \quad x \in \mathbb{R}, n \in \mathbb{N} .
$$

The function $q$ satisfies the quadratic functional equation, which implies

$$
|h(x)+n q(x)| \leq k|x|+\frac{1}{n} 2 \alpha, \quad x \in \mathbb{R}, n \in \mathbb{N},
$$

Hence $q(x)=0, x \in \mathbb{R}$ and

$$
|h(x)| \leq k|x|+2 \alpha, \quad x \in \mathbb{R} .
$$

It follows that, the additive function $h$ is bounded in the neighborhood of 0 , and consequently $h(x)=a x, x \in \mathbb{R}$ for some constant $a \in \mathbb{R}$. Thus

$$
|f(x)-a x| \leq k|x|, \quad x \in \mathbb{R}
$$

which gives

$$
\left|\frac{f(x)}{x}\right| \leq k+|a|, \quad x \in \mathbb{R} \backslash\{0\} .
$$

Let $N$ be such that $N \alpha>k+|a|$ and take an $x \in\left(0, \frac{1}{2^{N-1}}\right)$. Then $2^{n} x \in(0,1)$ for $n=0,1, \ldots, N-1$ and

$$
f(x)=\sum_{n=0}^{N-1} \frac{\phi\left(2^{n} x\right)}{2^{n}}+\sum_{n=N}^{\infty} \frac{\phi\left(2^{n} x\right)}{2^{n}}>N x \alpha
$$

so

$$
\frac{f(x)}{x}>N \alpha>k+|a|,
$$

which is contrary to (11). 
For $p=2$ we have the same example like in the case of the quadratic equation (see [2]).

Example 8. Let $\phi: \mathbb{R} \rightarrow \mathbb{R}$ be defined as

where $\alpha>0$. Put

$$
\phi(x)= \begin{cases}\alpha, & x \in(-\infty,-1] \cup[1,+\infty), \\ \alpha x^{2}, & x \in(-1,1),\end{cases}
$$

$$
f(x)=\sum_{n=0}^{\infty} \frac{\phi\left(2^{n} x\right)}{4^{n}}, \quad x \in \mathbb{R} .
$$

Then $f$ satisfies

$$
|f(x+y)+f(x-y)-2 f(x)-f(y)-f(-y)| \leq 32 \alpha\left(|x|^{2}+|y|^{2}\right)
$$

and there do not exist a function $g: \mathbb{R} \rightarrow \mathbb{R}$ satisfying the Drygas equation and a constant $k \geq 0$ such that

$$
|f(x)-g(x)| \leq k|x|^{2}, \quad x \in \mathbb{R} .
$$

Open Access. This article is distributed under the terms of the Creative Commons Attribution License which permits any use, distribution, and reproduction in any medium, provided the original author(s) and the source are credited.

\section{References}

[1] Brzdȩk, J., Chudziak, J., Páles, Zs.: A fixed point approach to stability of functional equations. Nonlinear Anal. 74, 6728-6732 (2011)

[2] Czerwik, S.: On the stability of the quadratic mapping in normed spaces. Abh. Math. Sem. Univ. Hamburg 62, 59-64 (1992)

[3] Drygas, H.: Quasi-inner products and their applications. In: Gupta, A.K. (ed.) Advances in Multivariate Statistical Analysis, pp. 13-30. Reidel Publishing Company, Boston (1987)

[4] Ebanks, B.R., Kannappan, Pl., Sahoo, P.K.: A common generalization of functional equations characterizing normed and quasi-inner-product spaces. Can. Math. Bull. 35, 321-327 (1992)

[5] Forti, G.L., Sikorska, J.: Variations on the Drygas equations and its stability. Nonlinear Anal. 74, 343-350 (2011)

[6] Jung, S.-M., Sahoo, P.K.: Stability of a functional equation of Drygas. Aequ. Math. 64, 263-273 (2002)

[7] Sikorska, J.: On a direct method for proving the Hyers-Ulam stability of functional equations. J. Math. Anal. Appl. 372, 99-109 (2010)

[8] Smajdor, W.: On set-valued solutions of a functional equation of Drygas. Aequ. Math. 77, 89-97 (2009)

[9] Yang, D.: Remarks on the stability of Drygas' equation and the Pexiderquadratic equation. Aequ. Math. 68, 108-116 (2004) 
Magdalena Piszczek and Joanna Szczawińska

Institute of Mathematics

Pedagogical University

Podchorążych 2

30-084 Kraków, Poland

e-mail: magdap@up.krakow.pl;

jszczaw@up.krakow.pl

Received: July 1, 2013.

Accepted: October 13, 2014. 